\title{
Safety and Effectiveness of Transthoracic Core Needle Biopsy in a Newly Established Interventional Radiology Program in Tanzania
}

\author{
Erick M. Mbuguje ${ }^{10}$ Jared M. Alswang ${ }^{2}$ Ivan Rukundo ${ }^{1}$ Azza Naif ${ }^{1}$ Fabian M. Laage Gaupp ${ }^{3}$ \\ Vijay Ramalingam ${ }^{4}$ Murray Asch $^{5}$
}

\footnotetext{
${ }^{1}$ Department of Radiology and Imaging, Muhimbili University of Health and Allied Sciences (MUHAS), Dar Es Salaam, Tanzania

2 Harvard Medical School, Boston, Massachusetts, United States

${ }^{3}$ Department of Radiology and Biomedical Imaging, Yale School of Medicine, New Haven, Connecticut, United States

${ }^{4}$ Department of Radiology, Beth Israel Deaconess Medical Center, Boston, Massachusetts, United States

${ }^{5}$ Diagnostic Imaging, Lakeridge Health Corporation, Oshawa,

Ontario, Canada
}

Arab J Intervent Radiol 2021;5:82-87.

\begin{abstract}
Address for correspondence Erick Michael Mbuguje, MD, MMed, Muhimbili University of Health and Allied Sciences, UN Road, Dar Es Salaam, Tanzania (e-mail: ericmbuguje@gmail.com).
\end{abstract}

\section{Abstract \\ Keywords \\ - cancer \\ - core needle biopsy \\ - transthoracic}

Background Transthoracic core needle biopsy (TTCNB) became a routinely offered procedure in Tanzania in October 2018. This study evaluates the safety and effectiveness of establishing a TTCNB program in a resource-limited setting.

Methodology A single center, prospective, observational cohort study was conducted at Muhimbili National Hospital on 90 patients who underwent computed tomography-guided TTCNB from October 2018 to May 2021. Patient and procedural data, including demographic information, complications, pathology results, and clinical outcomes, were stored in a Research Electronic Data Capture (REDCap) database. Follow-up was conducted at 4 weeks postprocedure by phone. Descriptive analysis was performed using Statistical Package for Social Sciences.

Results A total of 90 patients underwent TTCNB. Seven samples were lost or never processed. In total, 68/83 (81.9\%) of processed samples were diagnostic, with $89.7 \%$ ( $n=61)$ classified as malignant and $10.3 \%(n=7)$ classified as benign. Overall, $82.4 \%$ ( $n=56)$ were classified as primary malignancies, $7.4 \%(n=5)$ as metastatic malignancies, $5.9 \%(n=4)$ as benign tumors, and $4.4 \%(n=3)$ as infectious. Reasons for nondiagnostic samples were crashed/insufficient samples in $53.3 \%(n=8)$ and nonspecific chronic inflammation in $46.7 \%(n=7)$. Minor (Society of Interventional Radiology [SIR] class A and B) complications occurred in 8 cases (8.9\%), while there was 1 (1.1\%) major complication (SIR class F). A total of $44 / 90$ (48.9\%) patients could be reached for follow-up at 4 weeks postprocedure. In addition, 31/44 (70.5\%) of these patients had a diagnosis of malignancy. Of these, 20 received chemotherapy, 8 died published online February 2, 2022
DOI https://doi.org/ $10.1055 / \mathrm{s}-0041-1742220$ ISSN $2542-7075$. (c) 2022. The Pan Arab Interventional Radiology Society. All rights reserved.

This is an open access article published by Thieme under the terms of the Creative Commons Attribution-NonDerivative-NonCommercial-License, permitting copying and reproduction so long as the original work is given appropriate credit. Contents may not be used for commercial purposes, or adapted, remixed, transformed or built upon. (https://creativecommons.org/ licenses/by-nc-nd/4.0/)

Thieme Medical and Scientific Publishers Pvt. Ltd., A-12, 2nd Floor, Sector 2, Noida-201301 UP, India 
prior to receiving any treatment, 2 declined any further medical or surgical intervention, and 1 was treated with surgical excision and adjuvant chemotherapy.

Conclusion Although recently introduced in Tanzania, TTCNB has been performed with $81.9 \%$ diagnostic accuracy and a complication rate comparable to existing literature.

\section{Introduction}

The burden of cancer is rising in Africa. Between 2010 and 2030, the number of new cases and deaths from cancer is expected to double in the continent. ${ }^{1}$ While accounting for only $4.5 \%$ of new cancer cases worldwide, Africa made up $7.3 \%$ of cancer deaths globally, illustrating marked regional inequities in outcomes. ${ }^{2}$ In 2012, there were 39,300 new cases and 37,700 deaths from lung cancer reported in Africa. ${ }^{3,4}$ Although the reasons behind poor cancer outcomes in the continent are multifactorial, late-stage presentations and inadequate diagnostics have contributed significantly. ${ }^{4}$ Further, the lack of access to proper diagnostics not only worsens outcomes, but also serves as a barrier to proper surveillance. As a result, improving diagnosis has been identified as a critical and addressable way to reduce cancer morbidity and mortality. ${ }^{3}$

Transthoracic core needle biopsy (TTCNB), a first-line diagnostic tool in the diagnosis of benign and malignant thoracic lesions, including primary bronchogenic carcinomas, lung metastases, thoracic sarcomas, and lymphomas, was first formally offered in Tanzania in October 2018 following the launch of the nation's first interventional radiology (IR) service and training program at Muhimbili National Hospital (MNH). ${ }^{5}$ With the introduction of this new diagnostic tool in Tanzania, there is substantial promise to mitigate the nation's increasing burden of disease from thoracic cancers through improved diagnostic capabilities. The purpose of this study is to evaluate the feasibility and safety profile of TTCNB at MNH, providing a model for the implementation and use of TTCNB in similarly resourcelimited settings globally.

\section{Materials and Methods}

A single center, prospective, observational cohort study was conducted at MNH on 90 patients who underwent TTCNB from October 2018 to May 2021. This study received ethics approval by the hospital institutional review board. MNH is a tertiary health facility that serves as the national referral hospital for Tanzania. It is the largest hospital in Tanzania with approximately 3,000 inpatient beds and serves as the main research and training center for Muhimbili University of Health and Allied Sciences.

During the study period, 21 teams from the U.S.-based organization Road2IR, each including a fellowship-trained interventional radiologist, travelled to Tanzania on 2-week trips to train IR fellows at MNH. All TTCNBs were performed by Tanzanian IR fellows under the supervision of visiting faculty. At MNH there is not an existing protocol for the diagnosis of lung lesions, and as a result the majority of patients were referred to the IR service for biopsy by various departments throughout the hospital either after inconclusive pathology from fine-needle aspiration (FNA), the institute's previous first-line diagnostic approach, or based on clinical and/or radiological suspicion of thoracic malignancy. Patients were selected for TTCNB based on clinical presentation, imaging (computed tomography [CT], magnetic resonance imaging, ultrasound), and laboratory values. All patients who underwent TTCNB at MNH during the study period were included in this study.

Procedures were performed using CT-guidance and coaxial technique. 20-18G needles were used to extract tissue samples. Tru-Cut, full-core biopsy, screw, and helical needle types were all used during this study and were selected based on availability, as well as size, location, and depth of the lesion. Two to three tissue samples were taken during each procedure. Puncture-site bleeding was controlled by manual compression, and if unsuccessful with gelfoam, autologous blood clots, and/or saline to seal the biopsy needle tract.

Following the procedure, patients who underwent CTguided TTCNB were immediately evaluated for complications by thoracic CT. All patients were then transferred to the recovery room for 1 to 2 hours of clinical observation, pain evaluation, and monitoring of vital signs. A chest radiograph was obtained 1-hour postprocedure in all patients with suspected pneumothoraces. If there was no evidence for pneumothorax on chest radiograph and the patient was otherwise asymptomatic, the patient was discharged to the ward (if inpatient) or home (if outpatient). At discharge, patients were provided instructions on postprocedural care, potential late complications and their presenting symptoms, and when and how to seek further care. ${ }^{6,7}$ Patients were referred to their primary physicians for follow-up on the results.

Demographics, procedural information, and outcomes were recorded prospectively in a REDCap (Research Electronic Data Capture) database. Missing information was obtained through manual chart review of the hospital's electronic medical record. Descriptive statistical analysis was performed using Statistical Package for Social Sciences (SPSS).

\section{Results}

A total of 90 patients were included in this study. The mean age of study participants was 51.9 years (range: 10 months to 


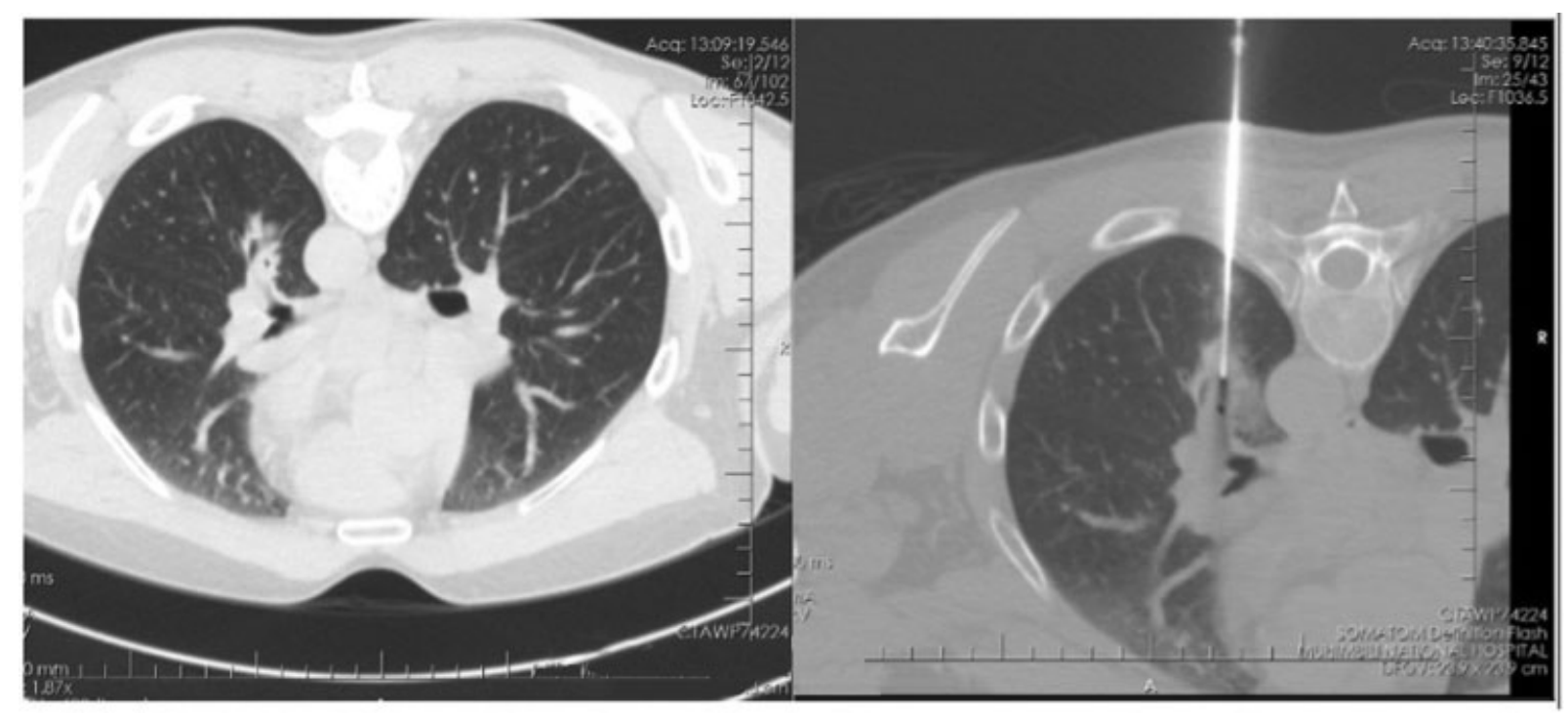

Fig. 1 CT-guided transthoracic core needle biopsy taken from a 53-year old male with confirmed small-cell lung carcinoma. CT, computed tomography.

86 years). In total, $57.8 \%(n=52)$ of study participants were male and $42.2 \%(n=38)$ were female. The vast majority (92.2\%) of referrals for TTCNB came from various departments within MNH: Surgery: $52.2 \%(n=47)$; Internal Medicine: $27.8 \%(n=25)$; Oncology: $5.6 \%(n=5)$, Pediatrics $3.3 \%$ $(n=3)$; General Practitioner 2.2\% $(n=2)$, and Nephrology $1.1 \%(n=1)$, while $7.8 \%(n=7)$ of referrals came from outside hospitals. A total of 85 patients were referred for suspicion of a thoracic malignancy and 5 for either suspicion of infection and malignancy. In addition, $70 \%(n=63)$ of biopsies were performed on an outpatient basis, while $30 \%(n=27)$ of biopsies were performed on inpatient basis. Tissue biopsy sites included: lung $(n=66)$, mediastinum $(n=20)$, pleura $(n=2)$, and chest wall $(n=2)$. All procedures were done under CT guidance. Of the 90 tissue samples extracted, 7 were lost or never processed and were excluded from further analysis. From the 83 samples that were processed, $81.9 \%$ $(n=68)$ were diagnostic ( - Fig. 1$)$. Diagnostic samples were malignant in $89.7 \%(n=61)$ and benign in $10.3 \%(n=7)$ of cases. Among the nondiagnostic samples reasoning included crashed/insufficient sample $53.3 \%(n=8)$ and nonspecific chronic inflammation $46.7 \%(n=7)$ (-Fig. 2 ).

Malignant lesions were further classified as primary bronchogenic carcinoma in $67.2 \%(n=41)$, metastatic disease in $8.2 \%(n=5)$, thymoma in $8.2 \%(n=5)$, sarcoma in $6.6 \%$ $(n=4)$, lymphoma $4.9 \%(n=3)$, and unspecified carcinoma in $4.9 \%(n=3)$. Benign lesions were further classified as tuberculosis in $42.9 \%(n=3)$, lung neurofibroma in $28.6 \%(n=2)$, ganglioneuroma in $14.3 \%(n=1)$, and sarcoidosis in $14.3 \%$ $(n=1)$.

The time to obtain a pathology report varied from 1 day to more than 30 days. The majority of results were available between 1 to 2 weeks from the day of the procedure: 51.8\% $(n=43)(-$ Table 1$)$.

Minor complications (Society of Interventional Radiology [SIR] class A and B) occurred in eight cases (8.9\%) while a major complication occurred in one $(1.1 \%)^{8}$ All minor complications were pneumothoraces and were managed by air aspiration during the procedure. The one observed major complication (SIR class F) occurred 1 hour postprocedure from a hemopneumothorax that was unsuccessfully managed with chest tube thoracostomy and oxygen administration through a non-rebreather mask, resulting in expiration of the patient ( $\mathbf{- T a b l e ~} \mathbf{1}$ ).

In total, $44 / 90(48.9 \%)$ patients could be reached for follow-up at 4 weeks postprocedure. And 31/44 (70.5\%) of these patients had a diagnosis of malignancy. Of these, 20 received chemotherapy, 8 died prior to receiving any treatment, 2 declined any further medical or surgical intervention, and 1 was treated with surgical excision and adjuvant chemotherapy. Among patients with benign tissue results, all received appropriate medical treatment.

\section{Discussion}

Epidemiologic and clinical data on thoracic malignancies in Tanzania are limited; however, GLOBOCAN 2020 estimates a total of 829 new cases and 762 new deaths from lung cancer in Tanzania annually. ${ }^{9,10}$ Tanzania lacks a national population-based cancer registry and the overall dearth of proper disease surveillance and screening in the country suggests that these figures are likely deflated. ${ }^{11}$ The few existing facility-based studies on thoracic malignancies in Tanzania likewise do not provide a complete picture on the status of thoracic malignancies in the country. ${ }^{12}$ It is evident that the lack of diagnostic capability has made it impossible to even begin to define the state of thoracic malignancy in Tanzania and the region.

In Tanzania, the available tools for diagnosing lung cancers are limited. At MNH, historically all thoracic lesions were biopsied by FNA, surgical excision, or more recently flexible bronchoscopy. In contrast, the use of TTCNB for 


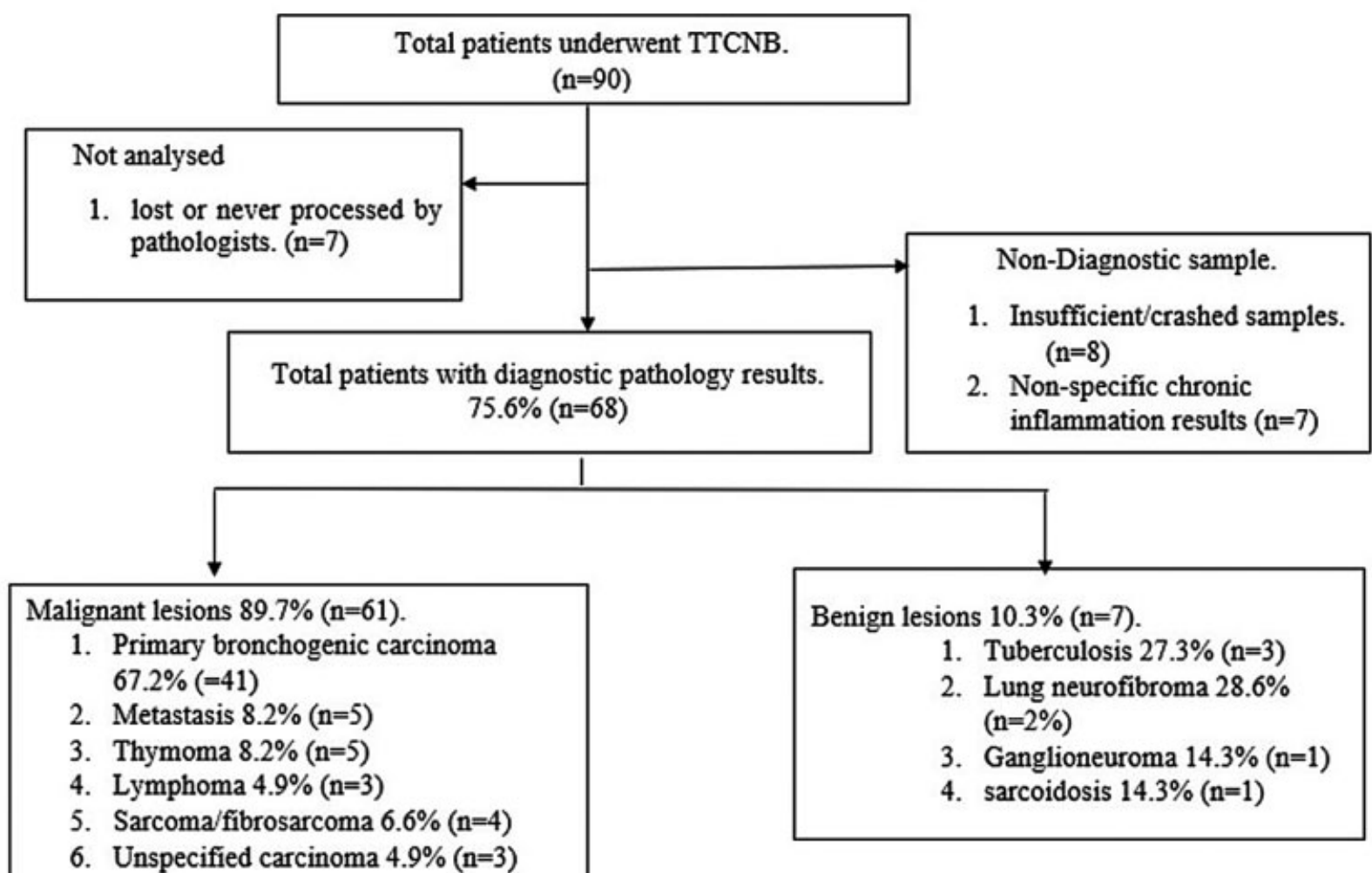

Fig. 2 Patient flow and pathology results.

diagnosing thoracic lesions is commonplace in high-income countries due to its efficacy in classifying the histology and molecular profile of thoracic lesions. ${ }^{13}$ Both TTCNB and FNA have been shown to be effective diagnostic tools in determining whether thoracic lesions are benign versus malig- nant. ${ }^{14}$ However, TTCNB is preferred due to its superior ability in classifying benign lesions and cancer subtypes, as well as its utility in biomarker analysis. ${ }^{13}$ With statistically similar complication rates between FNA and TTCNB, which include pneumothorax, pulmonary hemorrhage, and

Table 1 Percentage distribution of post-TTCNB complications

\begin{tabular}{|c|c|c|}
\hline & Frequency & Percentage \\
\hline Performed procedure & $N=90$ & $\%$ \\
\hline \multicolumn{3}{|l|}{ Post TTCNB complications } \\
\hline Yes & 9 & 10 \\
\hline No & 81 & 90 \\
\hline \multicolumn{3}{|l|}{ Complication distribution after TTCNB } \\
\hline Pneumothorax & 8 & 8.9 \\
\hline Death & 1 & 1.1 \\
\hline \multicolumn{3}{|l|}{ SIR complication categories } \\
\hline \multicolumn{3}{|l|}{ Minor complication } \\
\hline SIR class A-no therapy, no consequence & 6 & 6.7 \\
\hline $\begin{array}{l}\text { SIR class B-nominal therapy, no consequence } \\
\text { including overnight admission for observation only }\end{array}$ & 2 & 2.2 \\
\hline \multicolumn{3}{|l|}{ Major complication } \\
\hline SIR class F (death) & 1 & 1.1 \\
\hline
\end{tabular}

Abbreviations: SIR, Society of Interventional Radiology; TTCNB ,Transthoracic Core Needle biopsy. 
hemoptysis, the potential benefit of the introduction of TTCNB to a health system is clear. ${ }^{14}$

This study demonstrated that TTCNB is a safe and effective option for diagnosing thoracic lesions in Tanzania. While the accuracy rate for determining benign versus malignant lesions fell marginally below reported accuracy rates of TTCNBs in outside literature $(89.0-96.9 \%),{ }^{14}$ it is a stark improvement when compared with the estimated 65 to $85 \%$ accuracy rates of FNA without image guidance, previously the only available nonsurgical option for peripheral lesion biopsies in the region. Due to the ability to process tissue samples for both histologic and cytologic evaluation with core needle biopsies, the availability of this option with TTCNB further increases the potential for improving diagnostic yields of thoracic malignancies. ${ }^{15}$

Pneumothorax, the most common complication from CTguided TTCNB, is reported to occur in an average of $25.3 \%$ of patients across studies (-Fig. $\mathbf{3}$ ), a far higher rate compared with that observed in this study. ${ }^{16}$ Unfortunately, there was one recorded fatality in this study caused by a hemothorax in a patient with a suspected late-stage lung malignancy. This adverse outcome highlights the importance of meticulous preprocedural screening, as well as diligent postprocedural follow-up to timely manage any unforeseen complications that may develop. It should be noted that no other complications occurred aside from pneumothorax and the single hemothorax. This is especially significant when compared with the incidence of the second and third most common complications: pulmonary hemorrhage and hemoptysis, occurring on an average of 18.0 and $4.1 \%$, respectively. ${ }^{16}$ Aside from the one recorded SIR class $F$ complication (death), all other complications observed were of SIR class A or B (minor complications) and were adequately managed with no or nominal therapy. ${ }^{8}$ Overall, the low complication rate is indicative of the safety profile of TTCNB in Tanzania; however, it is evident that TTCNB has inherent risks that should not be minimized.

There are several limitations to this study. Due to the infancy of the IR program at $\mathrm{MNH}$, few patients have undergone a TTCNB and as a result the sample size of this study is small and continued data collection is needed both at $\mathrm{MNH}$ and other centers offering TTCNB to better generalize these findings. Further, this study was a prospective cohort study, and a randomized controlled trial is needed to compare TTCNB and FNA without image guidance in this setting. Additionally, because of hospital-wide issues with the electronic medical record and a lack of established methods of communication between patients and providers available at $\mathrm{MNH}$, reliable follow-up and systematic analysis of outcomes were difficult to achieve. Due to equipment limitations in the pathology department, molecular biomarkers and histological subtypes were not assessed from the tissue samples. As a result, the accuracy of biopsies was only analyzed based on overall diagnosis of the lesion. Currently, lung cancers are treated empirically at $\mathrm{MNH}$ with platinum-based regimens. However, as more targeted therapy treatment options become available in Tanzania, molecular biomarker and histological subtypes will become more relevant in this setting, furthering the benefit of TTCNBs.

The loss to follow-up in this study was notably high. The reason behind this is multifactorial, but is driven largely by financial limitations that include: incomplete record keeping, a lack of continuity in care, and a shortage of medical providers. Particular focus should be placed on improving
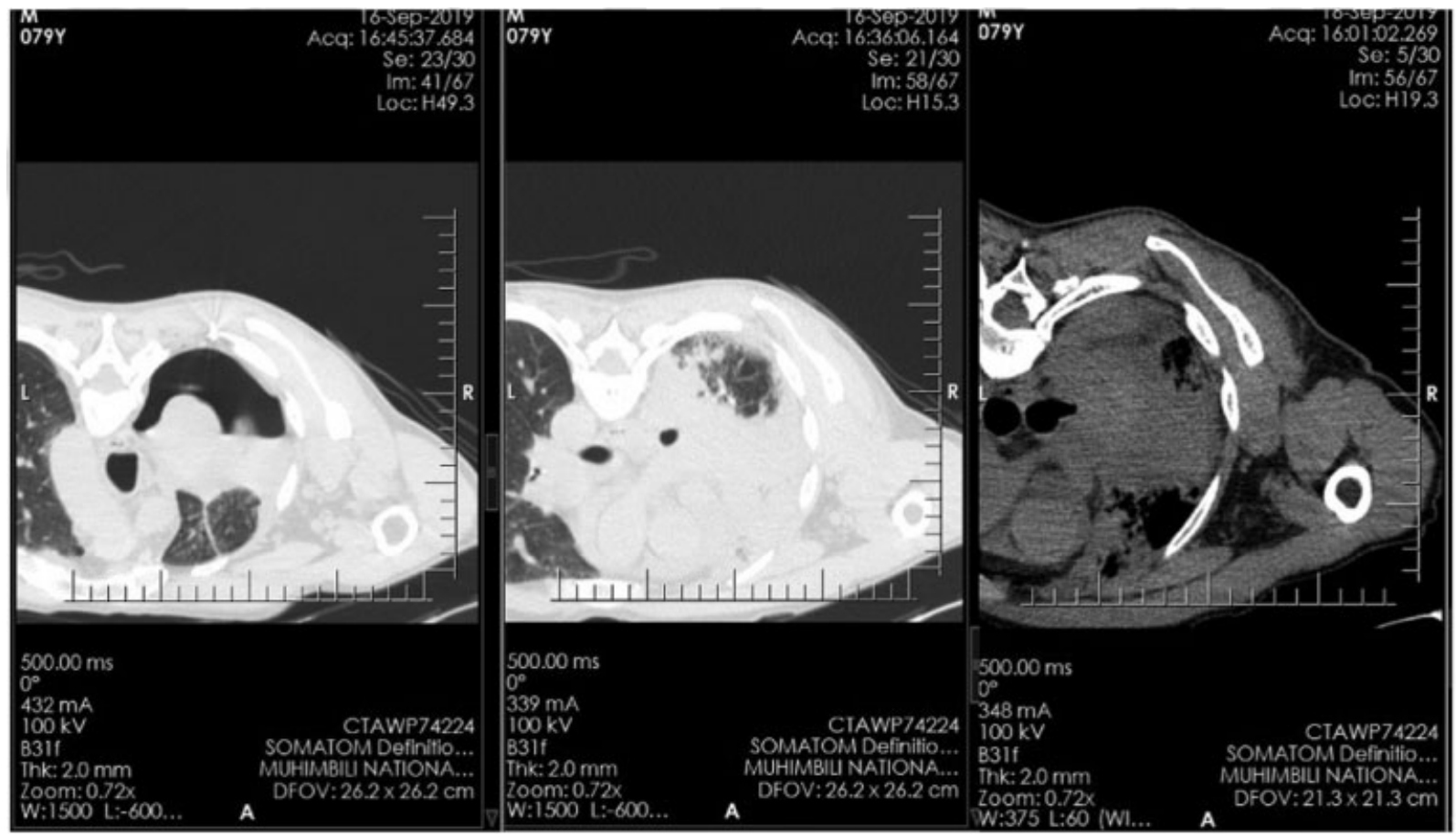

Fig. 3 Pneumothorax managed with needle aspiration following a CT-guided transthoracic core needle biopsy in a 79-year old male with a left lung mass and mild pleural effusion. Left: pre-needle aspiration (lung window); Middle: post-needle aspiration (lung window); Right: postneedle aspiration (soft tissue window). CT, computed tomography. 
systems that ensure proper patient selection criteria for TTCNB, timely awareness of diagnosis and its implications, and a thorough cost-benefit analysis of available treatment options. The overall poor outcome of patients enrolled in this study highlights the complex interplay of factors contributing to the low survival rates of thoracic cancers in subSaharan Africa, of which improved diagnostics solves only one piece of the puzzle. Additional emphasis should be placed on improving lung cancer screening as a whole. Shifting the trend to diagnosing lung cancers at earlier stages will greatly increase the potential impact that TTCNB may have, broaden available treatment options for patients, and ultimately reduce morbidity and mortality.

\section{Conclusion}

TTCNB has shown to be a both safe and effective method in diagnosing thoracic lesions in Tanzania with an acceptable diagnostic yield and rates of complications. Prior to the introduction of the IR service at MNH in 2018, image-guided biopsies were nonexistent in the country. Results from this study illustrate the opportunity that TTCNB presents in improving the diagnostics of thoracic lesions. As cancer rates continue to rise in Africa, so too does the need for improved diagnostic and treatment options available on the continent. Results from this study serve as a promising proof-of-concept for the widespread adoption of TTCNB and other interventional procedures in sub-Saharan Africa.

\section{Note}

Previous poster presentation: Society of Interventional Radiology (SIR) annual meeting, March 20-26, 2021.

This study received ethics approval by the hospital institutional review board (IRB).

\section{Competing Interests}

The authors have no competing interest to declare.

\section{Acknowledgments}

The authors appreciate the cooperation of all members of the Road2IR consortium for their endless contribution in the training program and for helping with the statistical analysis.

\section{References}

1 Jemal A, Bray F, Forman D, et al. Cancer burden in Africa and opportunities for prevention. Cancer 2012;118(18):4372-4384
2 Bahnassy AA, Abdellateif MS, Zekri AN. Cancer in Africa: is it a genetic or environmental health problem? Front Oncol 2020; 10:604214

3 Lubuzo B, Ginindza T, Hlongwana K. The barriers to initiating lung cancer care in low-and middle-income countries. Pan Afr Med J 2020;35:38

4 Cheng T-YD, Cramb SM, Baade PD, Youlden DR, Nwogu C, Reid ME. The International Epidemiology of Lung Cancer: latest trends, disparities, and tumor characteristics. J Thorac Oncol 2016;11 (10):1653-1671

5 Laage Gaupp FM, Solomon N, Rukundo I, et al. Tanzania IR initiative: training the first generation of interventional radiologists. J Vasc Interv Radiol 2019;30(12):2036-2040

6 Winokur RS, Pua BB, Sullivan BW, Madoff DC. Percutaneous lung biopsy: technique, efficacy, and complications. Semin Intervent Radiol 2013;30(02):121-127

7 Practice parameter for the performance of image guided percutaneous needle biopsy (PNB). 2018. Accessed May 13, 2021 at: https://www.acr.org/-/media/ACR/Files/Practice-Parameters/ pnb.pdf?la $=$ en

8 Cardella JF, Kundu S, Miller DL, Millward SF, Sacks DSociety of Interventional Radiology. Society of Interventional Radiology clinical practice guidelines. J Vasc Interv Radiol 2009;20(7, Suppl):S189-S191

9 Lyimo EP, Rumisha SF, Mremi IR, et al. Cancer mortality patterns in Tanzania: a retrospective hospital-based study, 2006-2015. JCO Glob Oncol 2020;6:224-232

10 Sung H, Ferlay J, Siegel RL, et al. Global Cancer Statistics 2020: GLOBOCAN estimates of incidence and mortality worldwide for 36 cancers in 185 countries. CA Cancer J Clin 2021;71(03): 209-249

11 Campbell JA, Soliman AS, Kahesa C, Harlow SD, Msemo D. Changing patterns of lung, liver, and head and neck non-AIDSdefining cancers relative to HIV status in Tanzania between 20022014. Infect Agent Cancer 2016;11:58

12 Ndilanha DA, Shayo GA, Hassan R, Byomuganyizi M, Lema LEK. Diagnoses from lung specimen collected through flexible bronchoscopy from patients in a tertiary hospital in Dar es Salaam Tanzania: a retrospective cross-sectional study. BMC Pulm Med 2019;19(01):214

13 Ocak S, Duplaquet F, Jamart J, et al. Diagnostic accuracy and safety of CT-guided percutaneous transthoracic needle biopsies: 14gauge versus 22-gauge needles. J Vasc Interv Radiol 2016;27(05): 674-681

14 Yao X, Gomes MM, Tsao MS, Allen CJ, Geddie W, Sekhon H. Fineneedle aspiration biopsy versus core-needle biopsy in diagnosing lung cancer: a systematic review. Curr Oncol 2012;19(01): e16-e27

15 Gasparini S. Histology versus cytology in the diagnosis of lung cancer: is it a real advantage? J Bronchology Interv Pulmonol 2010;17(02):103-105

16 Heerink WJ, de Bock GH, de Jonge GJ, Groen HJM, Vliegenthart R, Oudkerk M. Complication rates of CT-guided transthoracic lung biopsy: meta-analysis. Eur Radiol 2017;27(01):138-148 